\title{
Detection of a new, low-brightness supernova remnant possibly associated with EGRET sources
}

\author{
J. A. Combi ${ }^{1}$, G. E. Romero ${ }^{1}$, P. Benaglia ${ }^{1}$, and J. L. Jonas ${ }^{2}$ \\ 1 Instituto Argentino de Radioastronomía, C.C.5, (1894) Villa Elisa, Buenos Aires, Argentina \\ 2 Department of Physics \& Electronics, Rhodes University, Grahamstown 6140, South Africa \\ Received 19 September 2000 / Accepted 17 November 2000
}

\begin{abstract}
We report on the discovery of a shell-type supernova remnant in the southern sky. It is a large $\left(\sim 8^{\circ} \times 8^{\circ}\right)$, low-brightness source with a nonthermal radio spectrum, which requires background filtering to isolate it from the diffuse background emission of the Galaxy. Three 3EG $\gamma$-ray sources are spatially correlated with the radio structure. We have made 21-cm line observations of the region and found that two of these sources are coincident with HI clouds. We propose that the $\gamma$-ray emission is the result of hadronic interactions between high-energy protons locally accelerated at the remnant shock front and atomic nuclei in the ambient clouds.
\end{abstract}

Key words. ISM: supernova remnants - radio continuum: ISM - radiation mechanisms: nonthermal - gamma rays: observations

\section{Introduction}

The origin of cosmic rays (CRs) is a long-standing problem in contemporary astrophysics. It has been thought since the $1960 \mathrm{~s}$, on the basis of energetic considerations, that protons are accelerated up to energies of $\sim 10^{15} \mathrm{eV}$ in supernova remnants (SNRs) (Ginzburg \& Syrovatskii 1964). When first-order Fermi acceleration at shock fronts was established as an efficient mechanism for CR production (e.g. Bell 1978), SNRs became the most natural candidates for the accelerating agents of the bulk of galactic CRs. Multifrequency radio observations clearly show that SNRs accommodate a population of relativistic electrons with the expected energy distribution. However, there is at present not such a conclusive observational evidence for shock acceleration of protons and ions near SNRs.

High-energy $\gamma$-ray astronomy is the most appropriate tool to probe SNRs in the search for an observable signature of proton acceleration. The interactions between relativistic protons and ambient nuclei can produce neutral pions $\left(\pi^{0}\right)$ which decay to yield $\gamma$-ray emission that might be detected by instruments like the Energetic Gamma Ray Experiment Telescope (EGRET) on board the Compton satellite. Possible associations of $\gamma$-ray sources with SNRs have been claimed by several authors in the past (Pollock 1985; Combi \& Romero 1995; Esposito et al. 1996; Combi et al. 1998a). However, a leptonic origin of the $\gamma$-ray emission (e.g. Pohl 1996) cannot be ruled out in most of these cases, especially when relatively young remnants are considered.

In this paper we present results of a new observational study designed to clarify the problem of the origin of galactic CRs. Aharonian et al. (1994) and Aharonian \& Atoyan (1996) have pointed out that the presence of nearby clouds can increase the probability of detecting the nucleonic CR component locally accelerated in a SNR because the clouds act as targets for the relativistic protons, hence enhancing $\pi^{0}$ production and the resultant $\gamma$ ray luminosity. A large remnant in a cloudy medium may have several discrete $\gamma$-ray sources associated with it. The study of the spectra and fluxes of these $\gamma$-ray sources could provide information about the cosmic ray production at several different sites where the clouds are overtaken by the accelerated protons.

Some of the 170 unidentified 3EG $\gamma$-ray sources (Hartman et al. 1999) appear to occur in small clusters subtending less than $6^{\circ}$ in the sky at high galactic latitudes. We have searched the $408 \mathrm{MHz}$ all-sky survey (Haslam et al. 1981) for radio continuum emission towards the directions of those four clusters of high-latitude sources that are visible from the southern hemisphere. The EGRET confidence contours for the cluster consisting of 3EG J1834-2803, 3EG J1847-3219, and 3EG J18502652 correlate spatially with an extended shell-type radio source, which is masked by steep gradients in the diffuse galactic radiation. We have subsequently performed HI-line observations towards this large area in the Capricornus region, and have detected hydrogen clouds at the positions of two of the $3 \mathrm{EG}$ sources. Additional radio continuum data was provided by the Rhodes/HartRAO $2326 \mathrm{MHz}$ survey (Jonas et al. 1998). Having radio images at two widely-spaced frequencies allows the spatial distribution of the spectral index to be computed, hence establishing the nonthermal nature of the emission and 


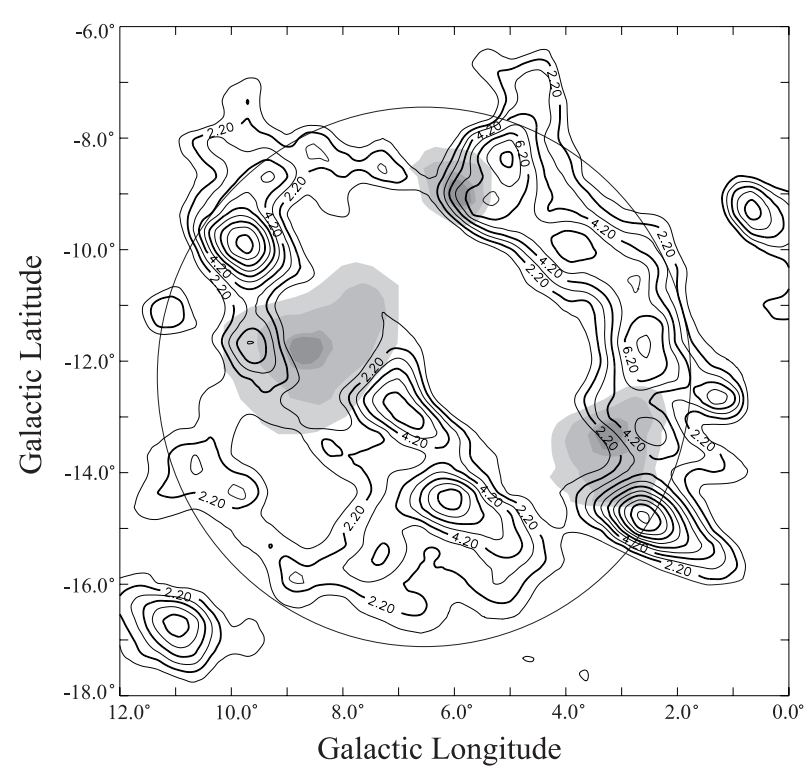

Fig. 1. Background filtered radio emission at $408 \mathrm{MHz}$ of the region surrounding the three $\gamma$-ray sources. Contours are labelled in steps of $1 \mathrm{~K}$ in brightness temperature, starting at $1.2 \mathrm{~K}$. The superposed grey-scaled levels represent the $99 \%$, $95 \%, 68 \%$, and $50 \%$ statistical probability that a $\gamma$-ray source lies within each contour according to the EGRET catalog (Hartman et al. 1999). HPBW $\sim 51^{\prime}$

revealing the energy distribution of the electronic component of the CRs at the SNR. In what follows we describe our data analysis procedures, new observations, and discuss the results of our study.

\section{Data analysis and new observations}

The detection of weak, extended radio sources is a difficult task due to the contamination produced by the diffuse background radiation in the radio images. Faint sources with diameters of several degrees can only be identified by using effective spatial filtering techniques applied to good-quality, single-dish data. In this study we have used data at $408-\mathrm{MHz}$ and $2326-\mathrm{MHz}$ from the radio surveys by Haslam et al. (1981) and Jonas et al. (1998), respectively. The angular resolutions (HPBW) of these surveys are $51^{\prime}$ $(408 \mathrm{MHz})$ and $20^{\prime}(2326 \mathrm{MHz})$. We have extracted maps of $12^{\circ} \times 12^{\circ}$ around the mean position of the $\gamma$-ray sources and have then used the background filtering method originally developed by Sofue \& Reich (1979) in order to emphasize the fine radio structure superposed on the diffuse galactic radio emission. This iterative procedure results in the elimination of diffuse sources with angular scales larger than a specified filtering beam (see Combi et al. 1998b for more details). This technique is very versatile, can be used at any galactic latitude, and is well-proven for a range of angular scales. It was recently used to discover the radio counterpart of the nearby SNR RX J0852.0-4622, which is contaminated by the bright, extended emission of the Vela region (Combi et al. 1999).

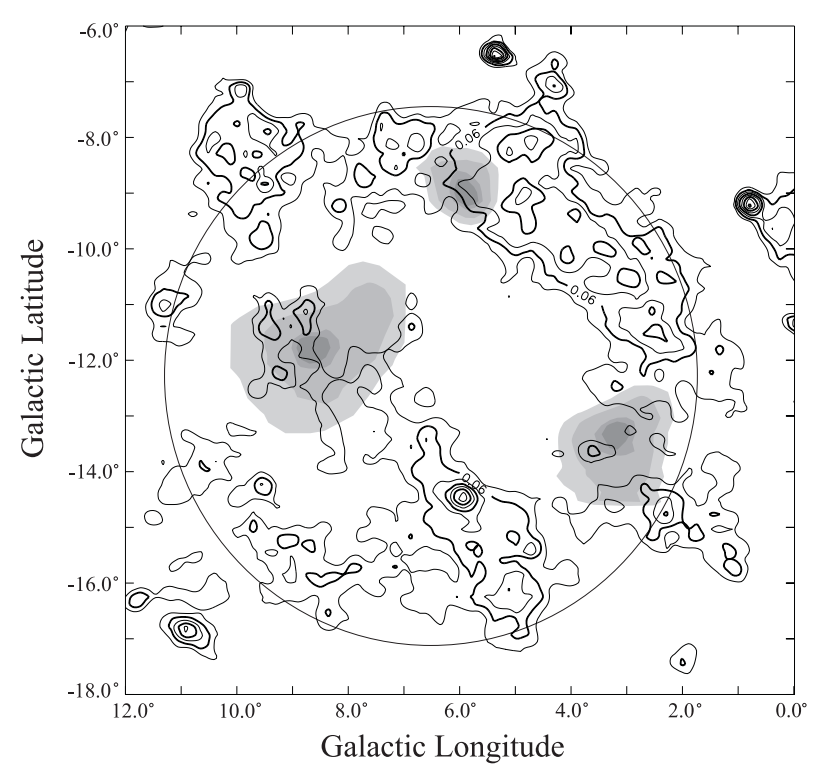

Fig. 2. Idem Fig. 1, but at $2.326 \mathrm{GHz}$. Brightness temperature contours are in steps of $0.03 \mathrm{~K}$, starting at $0.03 \mathrm{~K}$. HPBW $\sim 20^{\prime}$

When the $12^{\circ} \times 12^{\circ}$-maps are filtered, a steep gradient of emission shows up towards the galactic plane. In order to remove this residual background, we filtered a larger region of $20^{\circ} \times 24^{\circ}$, which includes the galactic plane. Then the smaller maps were extracted again from the filtered, larger map.

Figures 1 and 2 show the background filtered radio emission of the region under study at $408-$ and $2326-\mathrm{MHz}$, respectively. A filtering beam of $240^{\prime} \times 240^{\prime}$ was applied to both images (i.e. all emission structures with angular scales larger than $4^{\circ}$ have been suppressed in these images). The probability confidence contours of the $3 \mathrm{EG}$ sources have been superposed in the maps. A large $\left(\sim 8^{\circ} \times\right.$ $\left.8^{\circ}\right)$, shell-type source can be clearly seen at both radio frequencies. In order to determine the spectral index distribution of the shell emission we have convolved and retabulated the $2326-\mathrm{MHz}$ data to the same beam and tabular interval as the $408-\mathrm{MHz}$ map. Following Combi et al. (1998b), the spectral indices and the corresponding errors were estimated from these maps. Uncertainties from the filtering method also should contribute to the errors, but are difficult to estimate. We think that a conservative assumption here is to assume that the real errors double the formal ones. The spectral index distribution, which is shown in Fig. 3, is consistent with independent estimates obtained using a different filtering method developed by Jonas (1999).

Aharonian \& Atoyan (1996) emphasize that the existence of a potential CR accelerator, such as a SNR, is not a sufficient condition for the generation of detectable levels of $\gamma$-ray emission. Target nuclei are also required, so we have performed HI observations towards the area of interest in order to search for ambient atomic gas in the local neighbourhood of the SNR. The IAR 30-m single dish 
telescope at Villa Elisa, Argentina was used for these observations. The receiver was a helium-cooled HEMT amplifier with a 1008-channel autocorrelator at the backend, which has been used for similar purposes by Combi et al. (1998a,b). System parameters and additional details of the observational technique can be found in these papers.

The HI line was observed in hybrid total power mode and the sky was sampled on a $0.5^{\circ}$ rectangular grid. Each grid position was observed for $36 \mathrm{~s}$ with a velocity resolution of $\sim 1 \mathrm{~km} \mathrm{~s}^{-1}$ and a coverage of $\pm 450 \mathrm{~km} \mathrm{~s}^{-1}$. A set of HI brightness temperature maps $\left(\Delta T_{\mathrm{rms}} \sim 0.2 \mathrm{~K}\right)$ were built for the velocity interval $\left(-30 \mathrm{~km} \mathrm{~s}^{-1},+50 \mathrm{~km} \mathrm{~s}^{-1}\right)$, which is appropriate for this study. We adopted the following criteria to identify standard clouds in this velocity range: the maps should show a closed, simply connected structure (i.e. no shells or filaments were considered), with a clear maximum in brightness temperature, and an extension in velocity of at least $6 \mathrm{~km} \mathrm{~s}^{-1}$. There are six structures that satisfy this criterion in the region of interest. The two clouds, $\mathrm{C} 1$ centred at $(l, b) \sim\left(10^{\circ},-12^{\circ}\right)$ and $\mathrm{C} 2$ centred at $(l, b) \sim\left(5.5^{\circ},-8^{\circ}\right)$, are the stronger ones, with brightness temperatures of $\sim 45 \mathrm{~K}$, which are detected at a confidence level of $225 \sigma$. Figure 4 shows a series of $\mathrm{HI}$ brightness temperature maps where these clouds can be clearly appreciated. In Fig. 5 we present the integrated column density map for the relevant velocity interval with the 3EG sources superposed. Two of the sources (3EG J1834-2803 and 3EG J1850-2652) are positionally coincident with the clouds $\mathrm{C} 1$ and C2. Standard galactic rotation models (Fich et al. 1989) indicate that the remaining clouds, not shown in Fig. 4, are located at kinematic distances that are far too distant to be physically related to the continuum radio source, which due to its large size is constrained to be nearby. It is also interesting to notice that there is a local hole in the HI distribution at the approximate position of the center of the SNR.

\section{Main results}

The newly-identified extended shell-like radio source in the Capricornus region is centred at $(l, b) \approx$ $\left(+6.5^{\circ},-12.0^{\circ}\right)$. It has an integrated flux density of $\sim 180 \mathrm{Jy}$ at $408 \mathrm{MHz}$, once the contribution from background point sources has been removed. This extragalactic contribution is dominated by the sources PKS 1839-34, PKS 1848-338, and PKS 1853-300. Flux errors are difficult to estimate due to uncertainties related to the filtering technique, in such a way that the quoted flux should be considered only as a rough value.

The identification of the extended source as a new SNR is not only supported by its large size and shell-like morphology, but also by the spectral indices of the radio emission (see Fig. 3) which are clearly nonthermal with an average value of $\alpha \approx-0.68 \pm 0.15$, somehow steeper than those observed in typical of shell-type remnants (Green 2000). Notice, however, that the quoted errors are purely formal and, as it was previously mentioned, the real ones

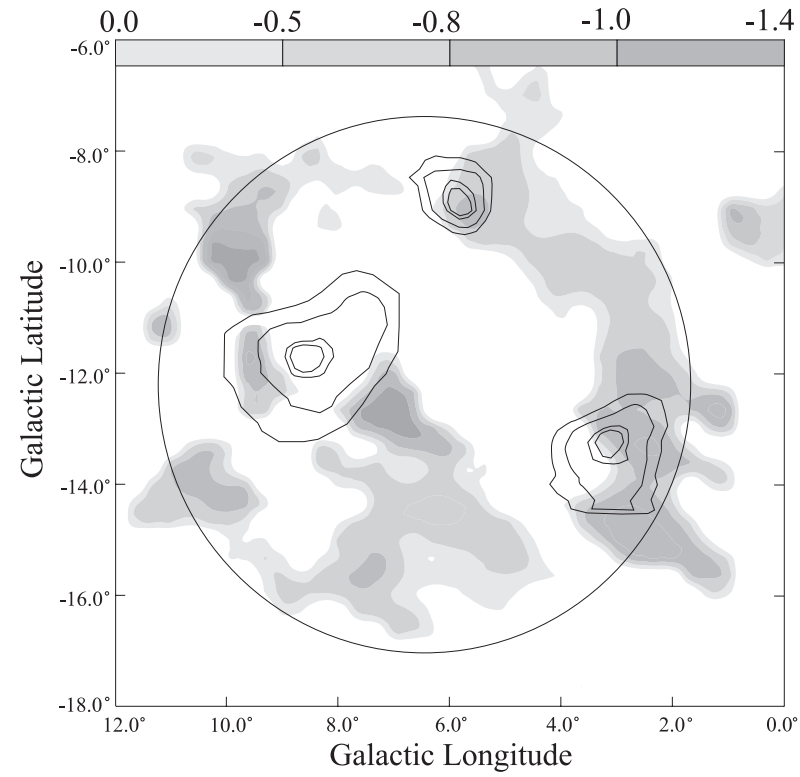

Fig. 3. Spectral index distribution computed between $408 \mathrm{MHz}$ and $2.326 \mathrm{GHz}$ for the region shown in Figs. 1 and 2. The EGRET probability contours are superposed

Table 1. Measured properties of the new SNR in Capricornus

\begin{tabular}{lc}
\hline Property & Value \\
\hline Galactic coordinates $($ center $)$ & $\left(+6.5^{\circ},-12.0^{\circ}\right)$ \\
Angular size $(\mathrm{deg} \times \mathrm{deg})$ & $8.0 \times 8.0$ \\
Flux density $(408 \mathrm{MHz})$ & $\sim 180 \mathrm{Jy}$ \\
Flux density $(2326 \mathrm{MHz})$ & $\sim 55 \mathrm{Jy}$ \\
Average spectral index & $-0.68 \pm 0.15^{1}$ \\
\hline
\end{tabular}

${ }^{1}$ Formal error.

could be significantly larger (perhaps around 0.3 ). The measured properties of this new Capricornus SNR are summarized in Table 1.

The distance to the remnant is uncertain, although its large size and high latitude suggest that it is nearby. A rough distance estimate of $d \sim 470$ pc can be obtained using the $\Sigma(D)$-dependence for low-brightness shell-like remnants proposed by Allakhverdigev et al. (1986), although this method alone is too dubious as to provide a reliable result. An independent upper limit of $700 \mathrm{pc}$ is provided by the relatively high value of $|b|$, if the source is assumed to be located within a galactic disk with a half-height of 150 pc. In addition, galactic rotation curve models (e.g. Fich et al. 1989) impose an upper bound of $\sim 500 \mathrm{pc}$ on the distance, assuming that the $\mathrm{HI}$ clouds $\mathrm{C} 1$ and $\mathrm{C} 2$ are physically related to the remnant.

A radius of $\sim 30 \mathrm{pc}$ is obtained for the SNR if a distance, not unreasonable, of $470 \mathrm{pc}$ is adopted, indicating that it is probably at the end of its adiabatic phase. Using standard Sedov solutions for an initial energy release of $\sim 10^{51} \mathrm{erg}$, we derive an age of $\sim 16500 \mathrm{yr}$, assuming an 


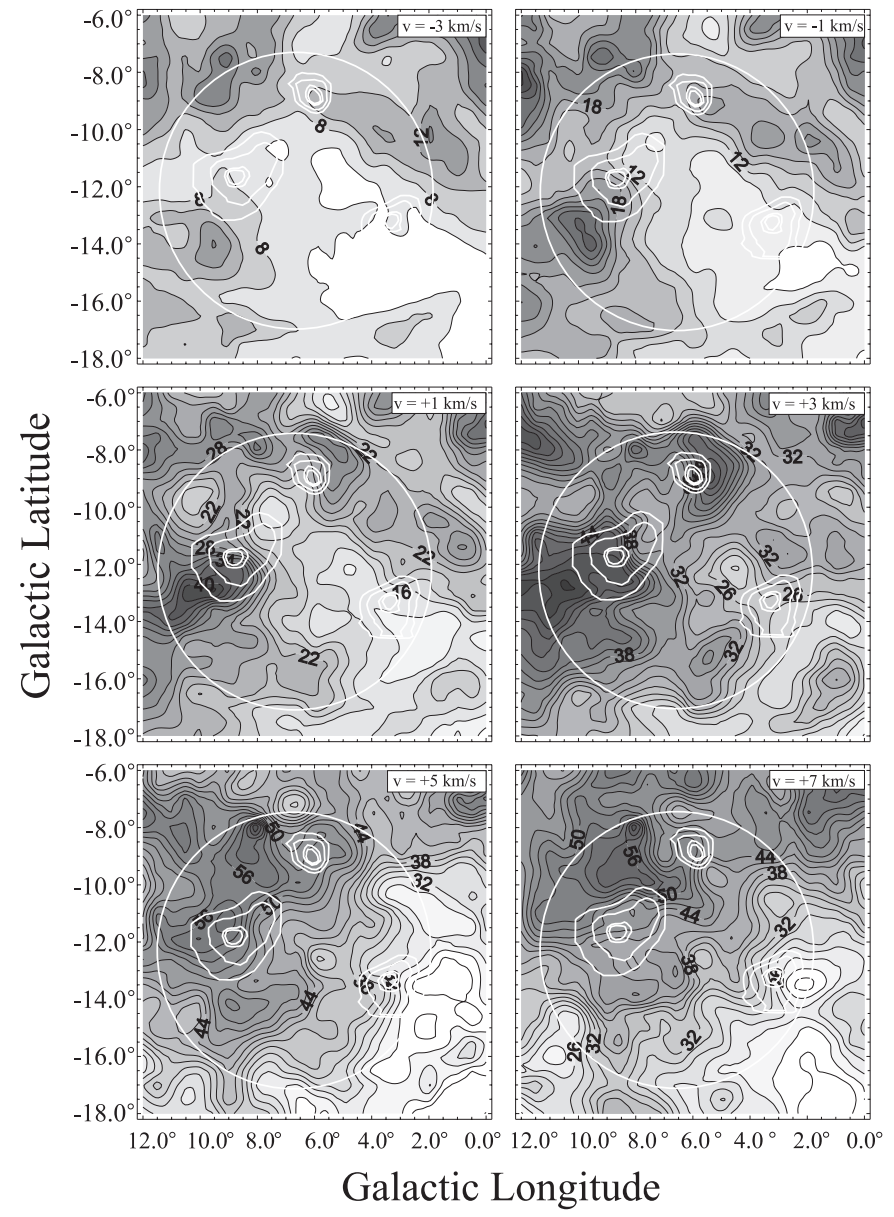

Fig. 4. HI brightness temperature channel maps (contour labels in $\mathrm{K}$ ) obtained for the velocity range $-3.0 \mathrm{~km} \mathrm{~s}^{-1}$ to $+7.0 \mathrm{~km} \mathrm{~s}^{-1}$. The supernova remnant boundary and the EGRET probability contours are superposed

average density of $n \sim 0.05 \mathrm{~cm}^{-3}$ for the intercloud medium at high galactic latitudes.

In Fig. 4 we show the HI clouds $\mathrm{C} 1$ and $\mathrm{C} 2$ using an ensemble of channel maps for the velocity interval -3 to $+7 \mathrm{~km} \mathrm{~s}^{-1}$, but one of the clouds is visible even beyond these velocity limits. $\mathrm{C} 1$ can be traced from -8 to $+2 \mathrm{~km} \mathrm{~s}^{-1}$ whereas $\mathrm{C} 2$ extends from -1 to $+5 \mathrm{~km} \mathrm{~s}^{-1}$. The cloud column densities were evaluated by integrating over these velocity intervals.

The integrated HI column density maps were used to obtain estimates for the total masses of the individual clouds. Using the adopted distance of $470 \mathrm{pc}$ we estimate masses of $\sim 1400 M_{\odot}$ and $\sim 1200 M_{\odot}$ for $\mathrm{C} 1$ and $\mathrm{C} 2$, respectively. The aggregate systematic error in these computed masses may be as large at $50 \%$. Dominant sources of error are uncertainties in the adopted distance, imperfect background subtraction, and possible existence of other (yet undetected) molecular species.

\section{Discussion}

The three $\gamma$-ray sources superposed with the SNR are classified as being possibly extended in the 3EG catalog, and none of them show variability. Variability is not expected if the $\gamma$-ray emission is related to the radio remnant.

The sources 3EG J1850-2652 and 3EG J1834-2803 are spatially coincident with the $\mathrm{HI}$ clouds $\mathrm{C} 1$ and $\mathrm{C} 2$, respectively. We suggest that the collective spatial coincidence of the $\gamma$-ray sources, HI clouds and the radio remnant provides evidence for the production of $\gamma$-rays by $\pi^{0}$-decay, where the pions result from proton-proton collisions at the $\mathrm{SNR} /$ ambient cloud interfaces.

In order to make quantitative estimates of the probability of chance association of the $\gamma$-ray sources in clusters, we have adapted the numerical code developed by Romero et al. (1999a) and used by Romero et al. (1999b) to study the positional association of unidentified EGRET sources with various populations of galactic objects. The code calculates angular distances between different kinds of celestial objects contained in selected catalogues, and establishes the level of positional correlation between them. Monte-Carlo simulations using large numbers of synthetic populations are then performed in order to determine the probabilities of pure chance associations. When generating synthetic populations of $\gamma$-ray sources the distribution in galactic latitude is constrained to be the same as that for the actual 3EG sources. This is necessary in order to obtain reliable results since the distribution of the $3 \mathrm{EG}$ sources is non-isotropic, with a strong concentration towards the galactic plane. The reader is referred to the paper by Romero et al. (1999b) for further details of the simulation code.

Using this code we determined that there are 54 unidentified 3EG sources (out of 116) that cluster within $6^{\circ}$ of each other at high latitudes $\left(|b|>5^{\circ}\right)$. When 1500 random populations of sources are generated (a larger number does not significantly change the results), the average number of sources in $6^{\circ}$ clusters is found to be $37.6 \pm 4.7$. This translates to a probability as low as $1.910^{-4}$ that all high-latitude $6^{\circ}$ clusters are chance events. For $|b|<5^{\circ}$ there are 47 sources out of 54 in $6^{\circ}$ clusters, whereas the simulations predict $34.6 \pm 3.4$ chance associations. The probability that all of the observed lowlatitude clusters are chance events is $1.510^{-4}$, which is similar to the value obtained for $|b|>5^{\circ}$. These simulations show that several apparent clusters of unidentified $\gamma$-ray sources are probably groups of physically related sources.

We shall now estimate the probability of finding a SNR by chance in the direction of a southern cluster of $\gamma$-ray sources. In order to do this, we need to know the chance probability of finding a shell-type, relatively large SNR overlapping with a circle of the cluster size in an arbitrary direction at intermediate (say $5^{\circ}<|b|<20^{\circ}$ ) galactic latitudes. There is just one of such SNRs in Green's (2000) catalog. Of course, many large and yet undetected remnants of low surface brightness should exist under the diffuse non-thermal galactic emission. A large-scale study with a systematic subtraction of the galactic background component of the southern radio sky at $2.326 \mathrm{GHz}$ by Jonas (1999) has recently shown that the number of these 
uncataloged SNRs in the relevant latitudes is about 17 . Since we have used data from the same Rhodes/HartRAO SKYMAP survey, we shall adopt this figure as a convenient estimate of the total number of remnants expected in the region of interest here. Then, the Poisson probability of finding one SNR overlapping with a cluster of 3 or more unidentified 3EG sources is $\sim 1.410^{-2}$.

The probability of chance association is further constrained if we consider that there are two clouds apparently coincident with two of the three $\gamma$-ray sources in the cluster for which the new SNR was found. The probability $p(n)$ that the line of sight along a distance $r$ will intersect with $n$ clouds is (Spitzer 1998):

$p(n)=\frac{(k r)^{n} \mathrm{e}^{-k r}}{n !}$,

where $k$ is the average number of clouds per $\mathrm{kpc}^{1}$. In the direction of the galactic plane $k \sim 6.2$ for standard clouds (Spitzer 1998). We found no published estimates of $k$ for relatively high latitudes, so we adopt the galactic plane value in order to obtain an upper limit to the probability. Using this equation and a distance range equal to the estimated diameter of the SNR (60 pc), we obtain that the chance probability of finding one or more clouds in each of two different lines of sight is 0.097 (about 10\%). Consequently, the probability that the observed configuration of SNR, $\gamma$-ray sources and HI clouds be the sole effect of chance superposition is about $1.310^{-3}$. This probability, although not overwhelming, suggests that the hypothesis of a multiple $\gamma$-ray source that could be causally related to the SNR deserves serious study in the light of its important potential implications.

There are mechanisms other than $p-p$ induced $\pi^{0}$ decay that may contribute to the $\gamma$-ray emission from SNRs. Inverse Compton (IC) scattering of relativistic electrons can boost ambient low-energy photon fields (such as the cosmic microwave background radiation, the diffuse galactic infrared/optical radiation, and the remnant's own photon fields) to $\gamma$-ray energies (e.g. Pohl 1996; Mastichiadis 1996). Bremsstrahlung emission from electrons in the shocked gas can also yield significant $\gamma$-ray luminosities (e.g. Blandford \& Cowie 1982). The relative contribution of each of these processes to the overall $\gamma$-ray flux from the remnant depends on factors such as the age of the remnant, the injected particle spectra, the ambient gas density, and the ratio of electrons to protons at high energies (e.g. Sturner et al. 1997; Gaisser et al. 1998).

In large and old remnants, such as the Capricornus remnant presented here, the IC boosting of diffuse background photons should dominate that of local radiation fields (Sturner et al. 1997). At high energies electrons cool more efficiently than protons by synchrotron and IC losses, therefore the electron-to-proton ratio in the source is ex-

\footnotetext{
1 This is actually an oversimplification because it ignores the fact that clouds form a power-law distribution of properties. The results, however, are not significantly changed in the present case, where massive clouds are considered.
}

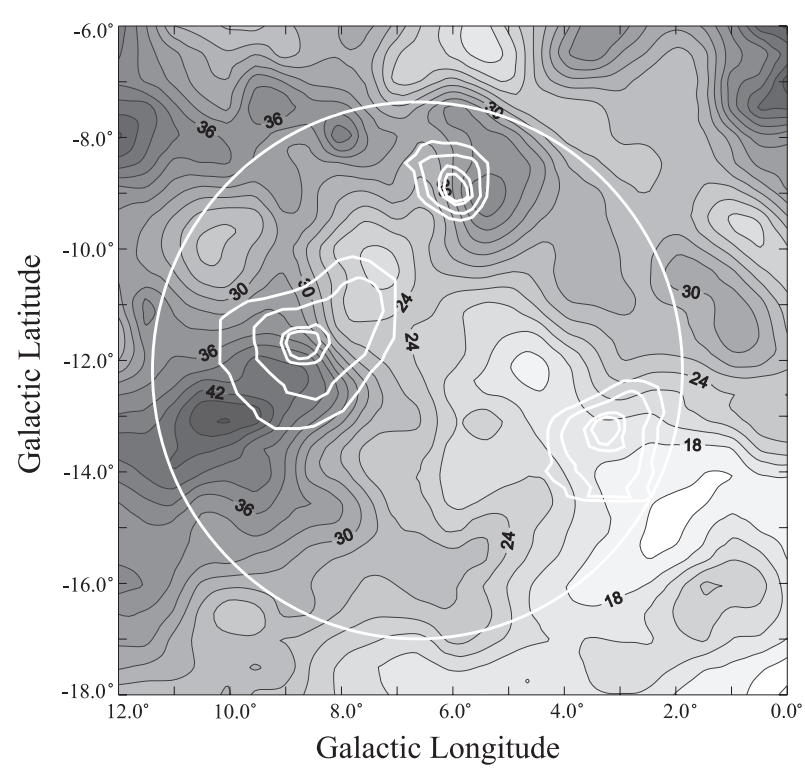

Fig. 5. A map of integrated column density of HI (velocity interval from $-3.0 \mathrm{~km} \mathrm{~s}^{-1}$ to $+5.0 \mathrm{~km} \mathrm{~s}^{-1}$ ) for the region of interest. Label units are $10^{19}$ atoms $\mathrm{cm}^{-2}$. EGRET probability contours are superposed

pected to be $\ll 1$, i.e. it would be similar to what is observed in the CRs near the Earth (see Aharonian et al. 1994, and especially Biermann \& Strom 1993 for additional arguments). IC radiation is therefore not expected to be dominant. Two factors indicate that the EGRET sources considered in this study are not predominantly due to IC scattering: (a) the detectable $\gamma$-ray emission is located towards regions where the observed radio emission (and hence the energy flux of relativistic electrons) is not particularly strong, and (b) two of the $\gamma$-ray sources occur towards HI clouds. If the HI clouds are sufficiently dense and the relativistic electron flux is high enough, then the observed $\gamma$-ray flux could be produced by bremsstrahlung interactions between the electrons and the ionized atoms in the compressed post-shock regions. This mechanism can, however, be discounted for our sources because both the cloud densities $\left(n \sim 10 \mathrm{~cm}^{-3}\right.$, inferred from the HI data) and the electron flux (estimated from the radio synchrotron emission) are far too low. The electron flux necessary to produce the observed $\gamma$-ray luminosity by bremsstrahlung mechanism would result in a synchrotron radio flux of about $100 \mathrm{Jy}$ at $1 \mathrm{GHz}$ for each cloud. This radio flux is two orders of magnitude greater than the value determined from the radio data for the regions occupied by the clouds in the remnant, and more than the flux density of the entire source at that frequency. We conclude, then, that $\pi^{0}$-decay is the dominant mechanism for $\gamma$-ray production in the new remnant. When an expanding SNR collides with a cloud, a fraction of the nuclear CR component is transported into the cloud by convection. This penetration produces a significant enhancement of the $\gamma$-ray luminosity because of the $\pi^{0}$-decays resulting 
from the $p-p$ interactions. The $\gamma$-ray flux expected at the Earth is:

$F(E>100 \mathrm{MeV})=\frac{1}{4 \pi} q_{\gamma} M_{\text {cloud }} d^{-2} m_{\mathrm{H}}^{-1}$,

where $q_{\gamma}$ is the $\gamma$-ray emissivity per $\mathrm{H}$ atom of the cloud of mass $M_{\text {cloud }}, d$ is the distance to the source, and $m_{\mathrm{H}}$ is the $\mathrm{H}$-atom mass.

Using the observed $\gamma$-ray fluxes from the C1 and C2 positions, the adopted distance of $470 \mathrm{pc}$, and our cloud mass estimates derived from the HI-observations, we find that there is an enhancement in the CR energy density in the clouds with respect to the value observed near the Earth (Dermer 1986). The enhancement factor is calculated to be $\sim 4.5$ for cloud $\mathrm{C} 1$ and $\sim 13$ for cloud $\mathrm{C} 2$, clearly indicating that protons are being accelerated at the remnant shock front.

Since at high energies the spectrum of $\pi^{0}$-decay $\gamma$ rays mimics the spectrum of the parent protons, we can infer from EGRET observations that at the position of C2 the proton spectrum is $N\left(E_{\mathrm{p}}\right) \propto E^{-2.6 \pm 0.2}$. This power-law index is similar to that of the electron component at the same position, as deduced from the radio brightness spectral index. The $\gamma$-ray spectrum towards $\mathrm{C} 1$ may be harder: $N\left(E_{\mathrm{p}}\right) \propto E^{-2.3 \pm 0.45}$. The radio emission from this region is too weak to provide a reliable electron spectral index for comparison. In both cases the proton spectral index is consistent with the theoretical value of $\Gamma=2.42$ that is expected from diffusive shock acceleration in shell-like remnants (Biermann \& Strom 1993; see also Gaisser et al. 1998, who consider a harder injection spectrum with $\Gamma=2.3$ ). Leakage from the galactic disk results in the steeper $E^{-2.75}$-spectrum observed near the Earth (Biermann \& Strom 1993). We estimate that the proton high-energy cutoff, considering the age of the SNR, should be about $80 \mathrm{TeV}$ (see Biermann \& Strom 1993 for the formulae).

\section{Conclusions}

We have discovered a new southern SNR in the Capricornus region. It is a large and faint shell-type nonthermal radio source which has till now been obscured by confusion with the diffuse galactic radiation. There are three $\gamma$-ray sources in the 3 EG catalog superimposed on this remnant. Two of these high-energy sources coincide with the positions of $\mathrm{HI}$ clouds detected at $21 \mathrm{~cm}$ in narrow velocity intervals. We have estimated the masses of the clouds and calculated the CR flux enhancement at the SNR, showing convincing evidence of proton acceleration at the shock front, as predicted by the theory. Interestingly, it is possible that the proton spectrum slope is not the same at all points in the remnant. The electron and proton components of the CR flux seem to display similar spectral slopes towards the single position where both spectra can be determined. Although the possibility of a pure chance association cannot be completely ruled out, it is clear that this southern cluster of $\gamma$-ray sources deserves more attention as a potential natural laboratory for CR studies. In particular, future X-ray and $\mathrm{TeV}$ observations of the new Capricornus SNR are required to provide the information necessary to determine the upper cutoffs in the spectrum of both the leptonic and the hadronic CR components within the source.

Acknowledgements. We are very grateful to Dr. D. F. Torres for his kind help with the numerical simulations and to an anonymous referee for useful comments. This research has been supported by the Argentine agencies CONICET (under grant PIP No. 0430/98) and ANPCT (PICT 98 No. 03-04881), as well as by Fundación Antorchas (funds granted to J. A. C. and G. E. R.). J. L. J. acknowledges financial support from the Joint Research Committee of Rhodes University.

\section{References}

Aharonian, F. A., Drury, L. O.' C., \& Völk, H. J. 1994, A\&A, 285,645

Aharonian, F. A., \& Atoyan, A. M. 1996, A\&A, 309, 917

Allakhverdiyev, A. O., Guseinov, O. H., Kasumov, F. K., \& Yusifov, I. M. 1986, Ap\&SS, 121, 21

Bell, A. R. 1978, MNRAS, 182, 147

Biermann, P. L., \& Strom, R. G. 1993, A\&A, 275, 659

Blandford, R. D., \& Cowie, L. L. 1982, ApJ, 260, 625

Combi, J. A., \& Romero, G. E. 1995, A\&A, 303, 872

Combi, J. A., Romero, G. E., \& Benaglia, P. 1998a, A\&A, 333, L91

Combi, J. A., Romero, G. E., \& Arnal, E. M. 1998b, A\&A, 333,298

Combi, J. A., Romero, G. E., \& Benaglia, P. 1999, ApJ, 519, L177

Dermer, C. D. 1986, A\&A, 157, 223

Esposito, J. A., Hunter, S. D., Kanbach, G., \& Sreekumar, P. 1996, ApJ, 461, 820

Fich, M., Blitz, L., \& Stark, A. A. 1989, ApJ, 342, 272

Gaisser, T. K., Protheroe, R. J., \& Stanev, T. 1998, ApJ, 498, 219

Ginzburg, V. L., \& Syrovastkii, S. I. 1964, The Origin of Cosmic Rays (Pergamon Press, NY)

Green, D. A. 2000, A Catalog of Galactic Supernova Remnants, Mullard Radio Astronomy Observatory, Cambridge, England, UK available on the World Wide Web at http://www.nrao.cam.ac.uk/survey/snrs/

Hartman, R.C., et al. 1999, ApJS, 123, 79

Haslam, C. G. T., Klein, U., Salter, C. J., et al. 1981, A\&A, 100, 209

Jonas, J. L., Baart, E. E., \& Nicolson, G. D. 1998, MNRAS, 297, 977

Jonas, J. L. 1999, Ph.D. Thesis, Rhodes University

Mastichiadis, A. 1996, A\&A, 305, L53

McLaughlin, M. A., Mattox, J. R., Cordes, J. M., \& Thompson, D. J. 1996, ApJ, 473, 763

Pohl, M. 1996, A\&A, 307, 57

Pollock, A. M. T. 1985, A\&A, 150, 339

Romero, G. E., Torres, D. F., Anchordoqui, L. A., et al. 1999a, MNRAS, 308, 659

Romero, G. E., Benaglia, P., \& Torres, D. F. 1999b, A\&A, 348, 868

Sofue, Y., \& Reich, W. 1979, A\&AS, 38, 251

Spitzer, L. 1998, Physical Processes in the Interstellar Medium, Wiley Classics Library (J. Willey \& Sons, NY), 155-156

Sturner, S. J., Skibo, J. G., Dermer, C. D., \& Mattox, J. R. 1997, ApJ, 490, 619 